\title{
Dynamic J-Point Elevation Associated with Epileptic Hemiplegia: The Osborn Wave of Todd's Paralysis
}

\author{
Erik O'Connell ${ }^{\mathrm{a}}$ Nevin Baker ${ }^{\mathrm{b}}$ Gopi Dandamudi \\ Steven Steinhubl ${ }^{b}$ \\ ${ }^{a}$ Internal Medicine, ${ }^{b}$ Cardiology, and ${ }^{\mathrm{C} C a r d i a c}$ Electrophysiology, Geisinger \\ Medical Center, Danville, Pa., USA
}

\section{Key Words}

Todd's paralysis · Osborn wave · J-wave - Epilepsy · Stroke · STEMI - Hemiplegia ·

Postictal · ACS

\begin{abstract}
This case report exposes a phenomenon which, although proposed, has not been described in clinical literature: transient postictal hemiplegia (Todd's paralysis) with concomitant electrocardiographic J-point deflection (Osborn waves). Although typically associated with hypothermia, a prominent J-wave on the electrocardiogram (ECG) results from a transmyocardial voltage gradient during ventricular repolarization. Rarely, the Osborn wave may be observed in a non-hypothermic setting such as hypercalcemia or cerebral hemorrhage. Transient postictal hemiplegia has been attributed to localized cerebral hypoperfusion resulting from motor cortex exhaustion following an epileptic seizure. The same central nervous system autonomic dysfunction has been theorized to produce subendocardial hypoperfusion with electrocardiographic change and cardiac troponin $T$ elevation. This is the first described ECG evidence of a dynamically displaced J-point in the setting of postictal hemiplegia.
\end{abstract}

\section{Background}

Typically described in association with hypothermia, a prominent J-wave on the electrocardiogram (ECG), referred to as the Osborn wave, results from a transmyocardial voltage gradient during ventricular repolarization [1]. Rarely, this phenomenon may be observed in a non-hypothermic setting including brain injury [2, 3]. Postictal epileptic hemiplegia, also known as 'Todd's paralysis', has been associated 
with localized transient cerebral hypoperfusion as a result of motor cortex exhaustion lasting an average of $15 \mathrm{~h}$ following a seizure [4]. The same central nervous system autonomic dysfunction can produce subendocardial hypoperfusion with electrocardiographic change and cardiac troponin T (cTnT) elevation [5].

\section{Case Report}

While shopping at his local market, a 75-year-old epileptic man had a witnessed loss of consciousness with urinary incontinence and subsequent postictal confusion. Paramedics noted leftsided upper and lower extremity hemiparesis with aphasia and he was taken to a local hospital. ECG performed within the first hour after the event revealed ST-segment abnormalities of V3-V6, with serum cardiac troponin I (cTnI) 0.01 (normal $0.00-0.40 \mathrm{ng} / \mathrm{ml}$ ) and no symptoms of cardiac ischemia (fig. 1). Tympanic temperature was $37.2^{\circ} \mathrm{C}$. Ionized calcium was $1.15(1.13-1.32 \mathrm{mmol} / \mathrm{l})$. The patient was transferred to a facility capable of emergent percutaneous coronary intervention for ST-elevation myocardial infarction (STEMI) and cardiology consultation was performed on arrival. Initial serum cTnT was $0.492 \mathrm{mg} / \mathrm{ml}$ (normal $<0.100 \mathrm{ng} / \mathrm{ml}$ ). Neuroimaging with non-contrast head CT and MRI did not reveal hemorrhage or focal ischemia. Signs of unilateral hemiplegia resolved within fifteen hours of seizure coinciding with resolved J-point displacement primarily in leads V3-V6 consistent with Osborne waves (fig. 2). A transthoracic echocardiogram (TTE) identified an akinetic cardiac apex with a normal base and reduced left ventricular systolic function. No segmental wall motion abnormality was identified to suggest ischemia of a single coronary territory. At its peak, cardiac biomarker elevation was attributed to CNS-mediated autonomic dysfunction due to flattened release of troponin [cTnT $0.687(<0.100 \mathrm{ng} / \mathrm{ml})$; CK 243 (30-259 U/l); MB fraction $24.1(0-8.9 \mathrm{ng} / \mathrm{ml})$; relative index $9.9(0-3.9 \%$ )] (table 1). Appropriate medical therapy was initiated and follow-up TTE at one month revealed normalization of cardiac function. This finding suggested resolution of a stressinduced cardiomyopathy.

\section{Discussion}

In this clinical vignette, hypoperfusion following a postictal surge of autonomic tone produced evidence of both cerebral and cardiac ischemia. Osborn waves have been described in other instances of cerebral injury such as trauma or hemorrhage; however, ECG evidence of a dynamically displaced J-point has not been previously described in the setting of postictal hemiplegia.

\section{Disclosure Statement}

The authors have no conflict of interest. 
Table 1. Cardiac troponin serum concentrations following epileptic seizure

\begin{tabular}{lllll}
\hline Elapsed time & $30 \mathrm{~min}$ & $2 \mathrm{~h}$ & $9.5 \mathrm{~h}$ & $18 \mathrm{~h}$ \\
\hline $\begin{array}{l}\text { cTnT } \\
\text { cTnI }\end{array}$ & 0.01 & 0.492 & 0.687 & 0.637 \\
\hline
\end{tabular}

* The normal range for cTnT and cTnI is $<0.010$ and $<0.40 \mathrm{ng} / \mathrm{ml}$, respectively. Note the flattened release of cTnT as postictal hemiplegia resolves.

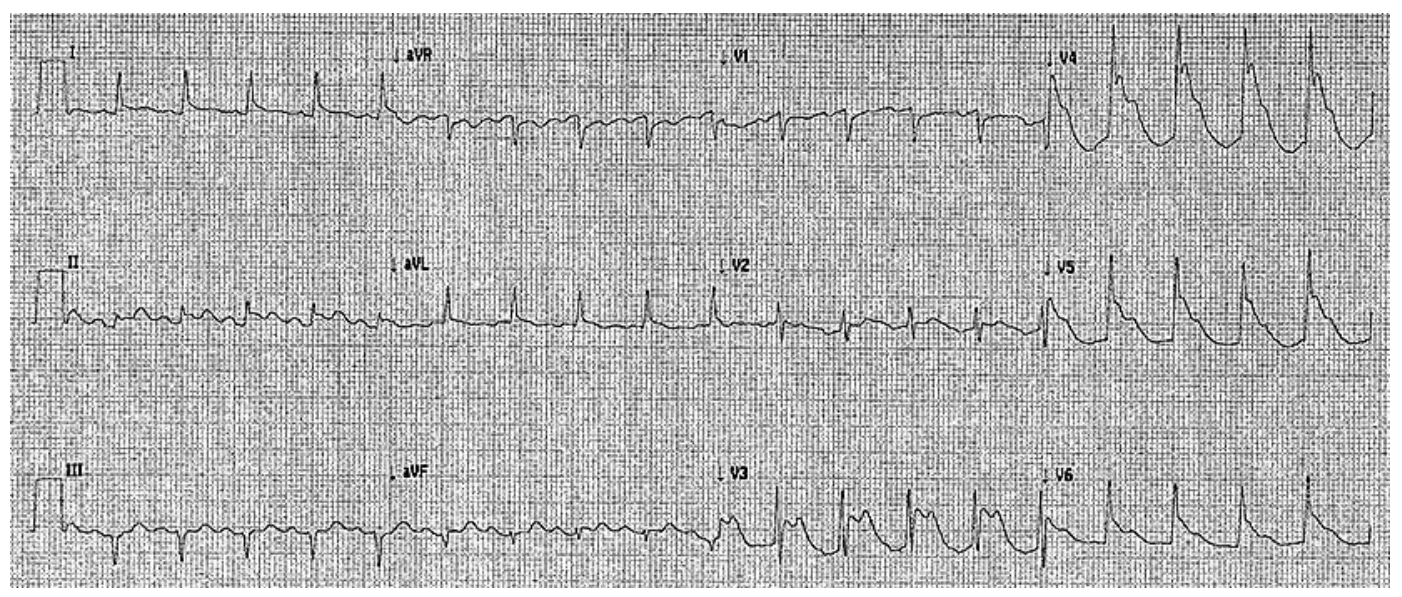

Fig. 1. ECG on presentation with J-point elevation in V3-V6. 
I
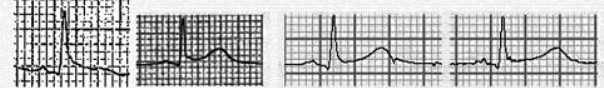

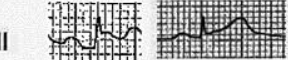

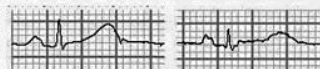

III

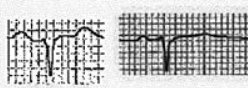

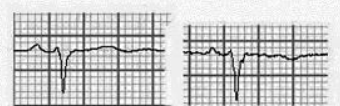

aVR

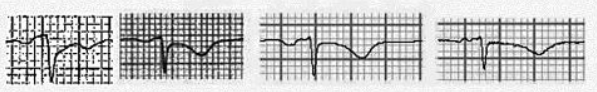

aVL
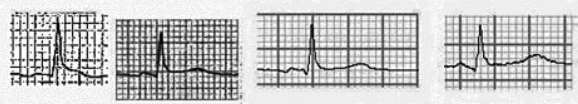

aVF 故赫期
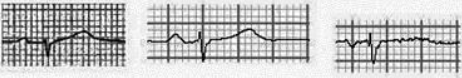
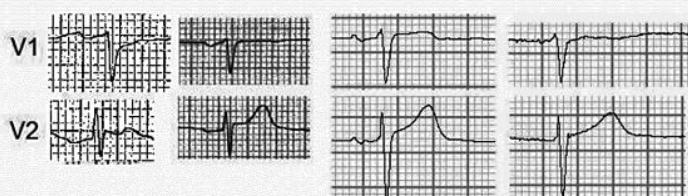

V3
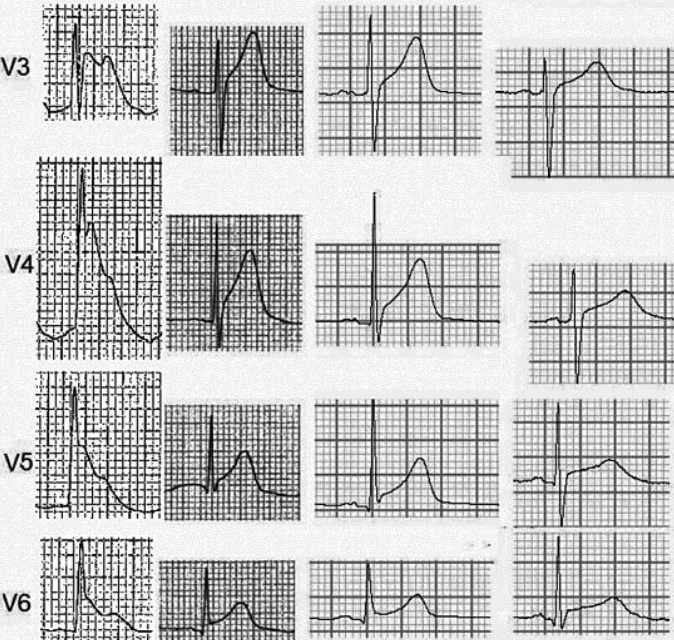

Fig. 2. J-point normalizes as hemiparesis resolves. ECG progression from presentation (approximately 45 min after seizure), prior to transfer (approximately 90 min after seizure), arrival (approximately $120 \mathrm{~min}$ after event), and the next morning (approximately $16 \mathrm{~h}$ after seizure).

\section{References}

1 Gussak I, Bjerregaard P, Egan TM, Chaitman BR: ECG phenomenon called the J wave. History, pathophysiology, and clinical significance. J Electrocardiol 1995;28:49-58.

2 Milewska A, Guzik P, Rudzka M, Baranowski R, Jankowski R, Nowak S, et al: J-wave formation in patients with acute intracranial hypertension. J Electrocardiol 2009;42:420-423.

-3 Yan GX, Antzelevitch C: Cellular basis for the electrocardiographic J wave. Circulation 1996;93:372-379.

4 Pearce JM: Robert Bentley Todd (1809-1860) and Todd's paralysis. J Neurol Neurosurg Psychiatry 1994;57:315.

5 Schuele SU: Effects of seizures on cardiac function. J Clin Neurophysiol 2009;26:302-308. 